\title{
Inpatient Rehabilitation Facility Including Rehabilitation distinct Part Units of a Hospital
}

National Cancer Institute

\section{Source}

National Cancer Institute. Inpatient Rehabilitation Facility Including Rehabilitation distinct Part Units of a Hospital. NCI Thesaurus. Code C99911.

A free standing rehabilitation center or a rehabilitation center within a general medical facility. (ACC) 